(Aus dem pharmakol. Institute und der Poliklinik fur Ohren-, Nasen- und Kehlkopfkranke an der Tniversitat zu Breslau.)

\title{
Beiträge zur Kenntniss der Wirkung des Chinins auf das Gehörorgan.
}

\author{
Erster Theil. \\ Sind die Wirkangen des Chinins am Gehörorgan auf Circulations- \\ störungen zurückzuführen? \\ Von
}

Dr. K. Wittmaack.

Unsere Kenntnisse über die Wirkung des Chinins auf das Gebörorgan stützen sich einerseits auf klinische Beobachtungen, andererseits auf experimentelle Untersuchungen am Menschen und am Thier.

So gross die Zahl der beobachteten Allgemeinintoxicationen mit Chinin ist, so zahlreich die easuistischen Mittheilungen uber Chininamaurose in der okulistischen Literatur auch sind, so spärlich ist die Zahl derjenigen Fälle, die vom otologischen Gesichtspunkte aus, wegen der bestehenden Störungen des Gehörorganes, genauer untersucht und beobachtet wurden. Ich sehe daher von einer genaueren Wiedergabe derjenigen Fälle, bei denen weder ein otoskopischer Befund erhoben, noch eine genauere Prufung des Hörvermögens unternommen worden ist, $a b$, da sie für eine Erkläung der Wirkung des Chinins auf das Gehörorgan wenig Wert baben. Zu ihnen gehören, soweit ich habe ersehen kónnen, sämmtliche Fälle von vorubergehender Schwerhörigkeit. Die über die Betheiligung des Gehörorganes bei diesen Fällen gemachten Angaben beschränken sich lediglich darauf, festzustellen, dass eine oft gleichzeitig mit einer Sehstörung einsetzende Taubheit bezw. Schwerhörigkeit, verbunden mit Ohrensausen und Schwindel, sich einstellte. Diese ging in der Regel schon innerbalb einiger Stunden bis Tage vollständig zurück. Die zu Grunde liegende Vergiftung war in bei Weitem der Mehrzahl der Fälle eine acute. Zurúckbleiben dauernder Schwerhörigkeit findet sich weit seltener erwähnt.

E. Pfluger, Archiv fux Physiologie. $B d 95$. 
$\mathrm{Zu}$ den Fällen dauernder Schwerhörigkeit gehören die in der specialistischen Literatur beschriebenen, von otiatrischer Seite aus beobachteten Fälle.

Soweit ich aus der mir zuganglichen Literatur ersehen kann, berichtet zum ersten Mal Ro o s a) uber einen Fall von dauernder Schwerhorigkeit nach Gebrauch grosser Chinindosen. Der Patient kam indessen erst, nachdem die Schwerhorigkeit schon Jahre lang bestanden hatte, in Behandlung. Ihre Deutung erscheint daher zweifelhaft, da Complication mit recidivirender Otitis externa und altem eingedickten Cerumenalpfropf (? Otitis med. des quamativa?) vorlag und eine genaue Hòrprufung nicht angegeben wurde.

Kirchner ${ }^{2}$ ) untersuchte - allerdings ebenfalls erst langere Zeit nach Einsetzen der Horstorung - Personen, die erklarten, von grossen Chiningaben taub geworden zu sein. Er fand hierbei wiederholt eine ausgesprochene Trubung des Trommelfelles mit weisser, sehnenartiger Verfarbung, die er als Residuen eines chronischen Entzundungsprocesses in der Paukenhohle auffasste. Die Horprüfung ergab Mangel der Perceptionsfahigkeit fur eine auf die Kopflnochen aufgesetzte tonende Stımmgabel und ferner mangelhafte Perception fur hohe Tone. Sie wies demnach auf eine gleichzeitige Labyrinthaffection hin. Deren Ursache sucht er in Entzundungsprocessen und hiernach zuruckgebliebenen Veranderungen.

$\mathrm{Schwabach}{ }^{3}$ ) beschresbt einen Fall, den er mehrere Monate nach Eintritt der Schwerhorigkeit in Behandlung bekam. Die Horstorung hatte sich im Anschluss an eine nicht naher erkannte fieberhafte Affection entwickelt, zu deren Bekampfung Chinin und Sahcylprapardte verordnet worden waren. Er konnte eine combinirte Stórung des schalleitenden (Veranderungen am Trommelfell) und des schallpercipirenden Apparates (Fehlen der Kopfknochenleitung, Herabsetzung der Perception fur die hohen Tone) feststellen. Gleichzeitig bestand Ohrensausen, Schwindel und Schmerzen im Ohr. Die Erkrankung des schalleetenden Apparates besserte sich wahrend der Bëhandlung, die des schallpercipirenden dagegen nicht.

Ueber einen ahnlichen Fall berichtet $S \operatorname{ch}$ hlling $\left.^{4}\right)$. $\cdot$ Perron $^{5}$ ) untersuchte nach mehreren Jahren emen Patienten, der drei Jahre lang im Anschluss am Malarıa mit grossen Chinndosen behandelt worden und allmahhich unter gleıchzeitig bestehendem Ohrensausen und Schwindel schwerhörig geworden war. Er schliesst aus dem Trommelfellbefund anf eine chronische Mittelohrerkrankung, stellte daneben aber, namentlich auf der starker befallenen Serte, eine Labyrinthaffection fest. Angaben uber eine genanere Horprufung fehlen. Die Behandlung war erfolglos.

1) Roosa, Transact. of the americ. otol. society. Fufth annual meeting 1872.

2) Kirchner, Ueber die Einwirkung von Chinin und Salicylsaure auf das Gebororgan. Berliner klin. Wochenschr. 1881 Nr. 49.

3) Schwabach, Ueber blelbende Storungen im Gehororgan nach Chıninund Salıcylsauregebrauch. Deutsche medic. Wochenschr. $1884 \mathrm{Nr} 11$.

4) Schilling, Munchner arztl. Intelligenzblatt $1883 \mathrm{Nr}$. 3.

5) Perron, Taubhert nach Chiningebrauch. Revue mens. de Laryng. etc. 1887 Nr. 11. 
Beitrage zur Kenntniss der Wirkung des Chinins auf das Gehororgan. 211

Einen weiteren Fall verdanken wir $\mathrm{D}_{a}$ bne ${ }^{1}$ ). Durch Gebrauch einer einmaligen sehr grossen Chinindosis war vor fünf Jahren eine totale Taubheit hervorgerufen worden und nach deren Rückgang eine dauernde Schwerhórigkeit zuruck geblieben. Auf Grund der Horprufung, der Intaktheit von Trommelfell und Tuben, halt Dabney das Labyrinth fúr den Sitz der Erkrankung.

Schliesślich berichtet noch $\mathrm{Moos}^{2}$ ) über einen Fall chronischer Chininvergiftung, die vor funf Jahren im Verlauf einer Malaria sıch entwickelt hatte und żu dauernder Schwerhorigkeit und Ohrensausen gefủhrt hatte. Die otoskopische Untersuchung (unveränderte Trommelfelle), ebenso wie die Hórprufung (Rinnescher Versuch positiv, starke Herabsetzung der Perception durch Knochenleitung und der hoben Tỏne) deuteten auf elne Affection des inneren Ohres.

Ziehen wir aus den angefuhrten klinischen Beobachtungen das Resumé, so müssen wir bezüglich der Fälle vorubergehender Schwerhörigkeit sagen: die Entwicklung der Hörstörungen unter starken subjectiven Gehörsempfindungen und Schwindel, ihr fast apoplectiformes Finsetzen bei schweren Fällen, der Rückgang bis zu vollständiger Wiederherstellung des Hörvermögens, hier meist verbunden mit einer analogen, nachweislich auf eine Affektion der Retina zuruckzufuhrenden Sehstỏrung, das alles weist entschieden auf eine Functionsstörung am inneren Ohre hin; wenn auch bei diesen Fällen keine genauere otologische Untersuchung hierfür als sicherer Beweis angefuhrt werden kann (vergl. z. B. Roberts ${ }^{3}$ ), Gradenig $0^{4}$ ), Uhth off $f^{5}$ ) u. a.).

Hiermit stimmen die Ergebnisse der genauer otologisch untersuchten Falle von $d a u$ ernder Schwerhörigkeit nach Chininintoxication insofern überein, als bei ihnen allen eine Affection des innern Ohres festgestellt worden ist. Ihre Zahl ist allerdings im Verhailtniss zur Zahl derjenigen Fälle, die nur von vorubergehenden Horstörungen befallen wurden, recht klein. Gemeinsam ist diesen, dass sich die Hórstörung im Anschluss an längeren Gebrauch von grossen Chinindosen und im Gegensatz zum plötzlichen Einsetzen der vorubergehenden Schwerhörigkeit bei schwerer acuter Vergiftung.

1) G. Dabney, Ein Fall von permanenter Taubheit, wahrscheinlich in Folge von Chrnin. Zeitschr. f. Ohrenheilk. Bd. 22 S. 33.

2) Moos, Handbuch der Ohrenheilkunde, herausgeg. von Schwartze, Bd. 1 s. 599. 1892.

3) Roberts, Lancet 1895. (Therapent. Monatshefte 1895 S. 633.)

4) Gradenigo, Handbuch der Ohrenheilkunde, herausgeg. von Schwartze, Bd. 2 S. 599.1892.

5) Uhth off; Berliner klin. Wochenschr. 1890 Nr. 25. 
mehr allmählich entwickelt hat. Bei einer Anzahl dieser Patienten bestand gleichzeitig eine Erkrankung des Mittelohres. Wenn man indessen erwägt, dass die Untersuchung bei allen diesen Fällen erst längere Zeit nach der Vergiftung vorgenommen werden konnte, so ist der Beweis eines directen Zusammenhanges dieser Mittelohrerkrankung mit der Giftwirkung sehwer mit Sicherheit zu erbringen. Es könnte sich sehr wohl um eine langsam progrediente chronische Mittelohrerkrankung gehandelt haben, die zur Zeit der Intoxication sich noch im Initialstadium befand, noch keine merklichen Hörstörungen verursachte und sich erst späterhin unabluängig von der durch die specifische Giftwirkung bedingten nervösen Schwerhörigkeit im Laufe der Jahre weiter entwickelte (Fälle von Perron, Kirchner).

Es könnte aber auch eine Complication der durch die Chininmedication bekämpften fieberhaften Erkrankung mit einem acuten Mittelohrleiden vorgelegen haben, die neben der durch Chininwirkung bedingten Labyrinthschwerhörigkeit eine als Residuum zu deutende Mittelohrschwerhörigkeit zuruck liess (Fall von Schwabach). Ja, selbst der Einwand, dass auch die Affection des inneren Ohres nicht auf Chininwirkung zurúckzuführen sei, sondern unabhängig von ihr sich entwickelt habe, lässt sich für keinen der beschriebenen Fälle sicher widerlegen, denn auch die nicht mit Mittelohraffection complicirten Fälle kamen erst längere Zeit nach Einsetzen der Schwerhörigkeit zur Beobachtung. Ein absolut einwandsfreier, d. h. kurz nach Einsetzen einer unzweifelhaft durch Chiningebrauch verursachten Hörstörung otologisch untersuchter und dann für längere Zeit weiter beobachteter Fall existirt meines Wissens überhaupt nicht. Wir mussen daher aus den klinischen Beobachtungen schliessen, dass durch eine Chininintoxication zwar momentan erhebliche Hörstörungen, die sogar zur Bezeichnung Chinintaubheit Berechtigung geben, verursacht werden können, dass aber in der Regel ein vollständiger Ruckgang erfolgt, und dass das Zurúckbleiben d a u ernd e r Schwerhörigkeit, falls diese uberhaupt auf eine Chininwirkung zurückzuführen ist, als Ausnahme zu betrachten ist.

Der Sitz der Schwerhorigkeit ist im innern Ohre zu suchen.

Ueber die Art der die Hörstörungen bedingenden Veränderungen geben uns indessen unsere klinischen Beobachtungen direct keinen Aufschluss. Es fehlt sowohl eine genaue otoskopische Untersuchung einer im Höhestadium der Intoxication befindlichen Person, als auch 
eine pathologisch-anatomische bezw. mikroskopische Untersuchung des Gehörorgans eines durch Chinin ertaubtēn bežw. der Intoxication erlegenen Individuums. Ueber den Sectionsbefund im Labyrinth eines nach Gebrauch von Natron salicyl ertaubten Mannes berichtet Mc. Bride ${ }^{1}$ ). Er fand in der Schnecke keine Veränderungen, dagegen war in den Bogengängen der perilymphatische Raum mit Bindegewebsbủndeln in verschiedener Dicke erfült.

Ein Analogieschluss aus der der Sehstörung zu Grunde liegenden Ischämie der Retina auf eine gleiche Circulationsstỏrung des häutigen Labyrinthes erscheint immerhin nicht unberechtigt.

Weitere Aufklärungen konnten experimentelle Untersuchungen geben. Bei der Untersuchung am Menschen konnte meist in der Höhe der verabreichten Dosen lange nicht so weit gegangen werden, als dies bei der Mehrzahl der klinisch beobachteten Intoxicationen der Fall war.

Die ersten Untersuchungen dieser Art stammen von $\mathrm{R}_{00 \mathrm{sa}^{2}}{ }^{2}$ ). Er fand bei drei Collegen, von denen zwei je 10, einer 15 Gran $(0,9 \mathrm{~g})$ Chinin nahmen, nach 1 bis 11/2 Stunden Röthung des Gesichtes, der Ohrmuschel, besonders des Ohrläppchens, Vollsein und Singen im Ohre, sowie stärkere Injection der Hammergriffgefảsse. Hieraus glaubt er sich zu dem Schluss berechtigt, dass analoge Verhältnisse im Labyrinth anzunehmen seien und demnach die specifische Chininwirkung auf Hervorrufung einer Congestion beruhe. Diese Angaben Roosa's wurden indess durch die sorgfältigen Untersuchungen Guder's ${ }^{3}$ ), die an zwölf Herren (meist Mediciner) vorgenommen wurden, widerlegt. Die verabreichte Dosis betrug in der Regel 1,0 g. Das Eintreten einer Hyperämie im äusseren Gehörgange und im Trommelfell, Injection der Hammergriffgefässe, konnten in keinem der Fälle, weder in der angegebenen Zeit, noch später wahrgenommen werden; im Gegentheil ging in fünf Fâllen eine vor dem Versuche sichtbar gewesene leichte Injection der Hammergriffgefässe in der gegebenen Zeit zurück. Subjective Gehörsempfindungen (Rauschen, Sausen, Klingen) traten durehgängig nach 1 bis 1 1/2 Stunden auf, während sich der ungünstige

1) Contrib. of the patholog. of the ear. Journ. of Anat. and Phys. vol. 4 .

2) Roosa, Experiments concerning defects of Quinine upon the ear. Transactions of the american otological Society vol. 2 part. I. 1875.

3) Guder, Experimente über die Chininwirkung, insbesondere anf das gesunde menschliche Gehororgan. Dissert. Berlin 1880. 
Einfluss des Chinins auf das Gehörvermögen nach 2 bis 3 Stunden geltend machte. Die Hörstörungen gaben sich vor Allem in der Herabsetzung der Perceptionsfähigkeit für feinere Geräusche (Taschenuhr) zu erkennen, „deren Höhepunkt mit dem tiefsten Stand der Temperatur im äusseren Gehörgange zusammenfiel ". Hörbeeinträchtigungen und subjective Geräusche gingen nach and nach innerhalb. von 12 Stunden vollständig zuruck. Gleichzeitig stellten sich bei acht Versuchspersonen Schwindelerscheinungen ein.

Mit diesen Ergebnissen stimmen die von Ferreri ${ }^{1}$ ) an 30 weiblichen, wegen Malaria in Behandlung stehenden Kranken yorgenommenen Untersuchungen gut ubberein. Sie bilden in zweierlei Richtung eine werthvolle Ergänzung. Erstens hat Ferreri sich nicht gescheut, verhältnissmässig recht grosse Chinindosen (bis zu $3 \div 4 \mathrm{~g}$ innerhalb von 2 Stunden!) zu verabreichen, die $\mathrm{zu}$ sehr deutlich nachweisbarer Beeinträchtigung des Hörvermögens fuhrten. Sodann wurde bei allen Untersuchten eine eingehendere Hörprüfung vorgenommen. ILetztere ergab neben einer mehr oder weniger starken. Herabsetzung der Perceptionsfảhigkeit für die Uhr eine beträchtliche Herabsetzung der Perceptionsfähigkeit fur die hohen' Töne und bei Zuleitung vom Knochen aus, dabei positiven Ausfall des Rinne'schen Versuches. Das Maximum der Hörstörung zeigte: sich nach 4 Stunden. Ausserdem bestanden laute subjective Gehörsempfindungen und Betäubungsgefühl. Die Wirkung einer Chinindosis von $3-4 \mathrm{~g}$ innerhalb 2 Stunden übertraf die von $2^{\circ} \mathrm{g}$ nicht in entsprechender Weise, so dass also die maximale Giftwirkung auf das Ohr beobachtet wurde. Besonders hervorhebenswerth erscheint noch die Thatsache, dass es trotz wiederholter Verabreichung dieser Dosen keinmal zu dauernden Hörstörungen kam, vielmehr sämmtliche Kranke wieder mit normalem Gehör entlassen wurden. Die Rückkehr des Hörvermögens erfolgte in der Regel innerhalb 24. Stunden nach Verabreichung der letzten Chinindosis.

Dasselbe Resultat erhielt $\mathrm{Moos}^{2}$ ) in einem Versuche.

In etwas anderer Art experimentirte $\mathrm{Schul}^{8}$ ) an zehn jingeren Medicinern. Er verabreichte das Chinin in sebr kleinen Dosen

1) Gherardo Ferreri, Sulle sesioni dell' orecchio dovute alla malaria. Firenze 1887.

2) Moos, a. a. O.

3) Schulz, Studien uber die Wirkung des Chinins beim gesunden Menschen. Virchow's Archiv Bd. 109 S. 21. 
Beitrage zur Kenntniss der: Wirkung des Chinins auf das Gehororgan. 21כ

$(0,005 \mathrm{~g}$ "bis $0,01 \mathrm{~g})$ mehrere Wochen hindurch täglich bis zu einem Gesammtverbrauch von $0,2 \mathrm{~g}$ bis $0,3 \mathrm{~g}$. Die hierbei boobachteten Störungen von Seiten des Gehörorganes waren verhältnissmässig gering. $6 \mathrm{mal}$ wurde uber meist nach wenigen Stunden wieder verschwundenes Ohrensausen geklagt; nur in einem Falle hielt dieses längere Zeit an, in der Regel am Abend wiederkehrend. Taubheitsgefuhl, das eben so schnell wie das Ohrensausen zuruck ging, wurde nur zwei Mal angegeben. Daneben wurden zuweilen Schmerzen stechender Art hinter dem Ohr und im Gehörgang angegeben, Trommelfelluntersuchungen und Hörprüfuug wurden nicht vorgenommen.

Die experimentellen Untersuchungen am Menschen lehren uns; dass gröbere, auf entzundliche Prozesse hindeutende Verảnderungen in der Paukenhöhle bei der Chininintoxication nicht constant nach ${ }^{*}$ zuweisen sind; denn selbst die Richtigkeit der Roosa'schen Beobachtung zugestanden, deutet sie doch nur auf leich te Circulations. stỏrungen bin, die zur Erklarung der intensiven Ohrgeräusche und der Herabsetzung des Hörvermögens, wie sie bei Chininintoxication beobachtet wird, nicht hinreichen. Sehen wir doch nach Manipulationen im Gehorgang (Ausspritzen von Cerumen u. s. w.) und vor, Allem. nach der Bougirung der Tuben zuweilen noch intensivere Röthungen am Trommelfell, ja sogar kleine Petachien auftreten, ohne dass irgend welche Hörstorungen sich, einstellen. In Uebereinstimmung mit den klinischen Beobachtungen deuten auch die experimentellen Untersuchungen am Menschen auf eine Storung in inneren Ohre hin. Besonders hrervorzuheben ist, dass diese, wie es auch in der Regel bei den klinisch beobachteten Chininintoxicationen der, Fall war, innerhalb verhältnissmassig kurzer Zeit (24 Stunden bis wenige Tage) vollständig zuruck zu gehen pflegt, trotz Anwendung so grosser Dosen, dass die maximale Giftwirkung auf das Ohr erzielt wurde (Ferreri). Sie dienen somit zu einer Unterstützung dęr Einwände, die gegen die Beweiskraft der Fälle von dauernder Schwerhörigkeit nach Chiningebrauch erhoben werden mussten. Ueber die Art der zu Grunde liegenden Veränderungèn geben sie uns, ebensowenig wie die klinischen Beobachtungen, Aufklärung. Der Schluss, Roosa's aus der von ihm beobachteten Hyperämie der Paukenhỏle, deren regelmässiges Vorhandensein, wie gesagt, von späteren Untersuchern nicht bestätigt worden ist, auf einen Congestionszustand im Labyrinth erscheint nicht gereehtfertigt. 
Bei dem Versuch, durch Experimente am Thier etwa der Hörstörung zu Grunde liegende pathologisch-anatomische Veränderungen aufzufinden, muss man sich von vornherein darüber klar sein, dass der Thierversuch nur als Ergänzung zur klinischen Beobachtung und experimentellen Vergiftung am Menschen dienen kann, und dass die etwa gefundenen Veränderungen mit Vorsicht und nur insoweit verwerthbar sind, als sie mit den klinischen Beobachtungen am Menschen uberein stimmen. Der Nachweis einer durch das Chinin verursachten entsprechenden Hörstörung beim Thier ist - höchstens mit Ausnahme der Hunde und Affen - sehr schwierig, ja fast unmöglich. Auch muss, da beim Menschen Störungen von Seiten des Gehörorganes constant auftreten, falls die dargereichten Dosen nicht allzu klein blieben, ein ebenso constantes Vorkommen der etwa beim Thier sich findenden Veränderungen gefordert werden, wenn sie zur Erklärung für die angenommene Hörstörung verwerthet werden sollen. Das gilt jedenfalls, wenn ein analoges Verhalten des thierischen Gehörorganes vorausgesetzt wird: kommen doch beim Thiere in der Regel sehr hohe, ja tödtliche Dosen zur Anwendung.

Die ersten experimentellen Untersuchungen am Thier stammen von Kirchner ${ }^{\mathbf{1}}$. Er vergiftete Kaninchen, Katzen, Hunde, Meerschweinchen und Mäuse mit grossen, meist tödtlichen Chinindosen. Sie wurden den Kaninchen, Katzen und Hunden durch die Schlundsonde, den Meerschweinchen und Mäusen durch subeutane Injection applicirt. Während die Hunde, Katzen, Mäuse und Meerschweinchen den verabreichten grossen Dosen innerhalb kurzer Zeit erlagen, zeigten sich die Kaninchen selbst verhältnissmässig grossen Dosen von $1,5 \mathrm{~g}$ Chinin muriat. gegenuber resistent. Sie wurden nach achttäơiger Futterung getödtet, doch fehlen leider Angaben daruber, auf welche Art dies erfolgte. Kirchner beschränkte sich zunächst nur auf makroskopisehe Untersuchung der herausgenommenen Schlafenbeine. Er fand hierbei bei sämmtlichen Thieren gleiche Veränderungen: Hyperämie und Ecchymosen der Paukenböhlenschleimbaut; letztere indessen, wie er selbst hervorhebt, nicht regelmässig auf beiden Seiten; ausserdem konnte er "einige Male" bei Hunden lebhafte Röthung und ein stark gefülltes, geschlängeltes Gefäss in der ersten Schneckenwindung beobachten. Er schliesst

1) Kirchner, a. a. 0 . 
Beitrage zur Kenntniss der Wirkung des Chinins auf das Gehörorgan. 217

hieraus, "dass das Chinin an wichtigen Theilen des Gehörorganes Veränderungen hervorzurufen vermag, die das Hörvermögen nicht bloss hochgradig benachtheiligen, sondern auch gänzlich zerstören können. Die Betheiligung des inneren Ohres - des Labyrinthes in seiner Gesammtheit an dem hyperämischen Zustande, der besonders in der Paukenhöhle stark ausgeprägt ist und zu Hamorrhagien führen kann, wird für lảngere Zeit nicht ohne schwere Schädigung der Endausbreitungen des Nervus acusticus ertragen werden". In Ergänzung dieser Untersuchungen berichtet er noch in einer späteren Mittheilung ${ }^{1}$ ) über die mikroskopische Untersuchung der Gehörorgane der mit Chinin gefutterten Thiere. Sie hat ergeben: "Dass sowohl in der Schnecke als auch in den halbzirkelformigen Canálen ausser bedeutender Fullung der Blutgefässe auch Extravasate an verschiedenen Stellen nachgewiesen werden konnten".

Analoge Resultate erhielt Ferreriª), der Katzen durch subcutane Injectionen von salzsaurem Chinin tödtete und die unmittelbar post mortem entnommenen Schläfenbeine mikroskopisch in Serienschnitten untersuchte. Er gibt an, fast constante Blutungen im Labyrinth und im Stamme des Nerv. acusticus gefunden zu haben.

Hierdurch schien die Annahme Ro os a's von einem Congestionszustande im Labyrinth eine neue wichtige Stütze gefunden zu haben. Es erscheint an sich zwar ganz plausibel, die in der Mehrzahl der Fälle schnell vorubergehende Schwerhörigkeit bei Chininintoxication auf eine einfache Hyperämie des Labyrinthes zuruckzufuhren, und fur diejenigen wenigen Fälle, bei denen bleibende Störungen restiren, eine so erhebliche Steigerung, auf Grund irgend einer Prädisposition, anzunehmen, dass es zu kleinen Zerreissungen der feinen Gefässwảnde und somit zu kleinen Hämorrhagien kommt. Die Resorption und Organisation der Hamorrhagien wurden dann zu den bleibenden Veränderungen führen können, die von Kirchner, Schwabach und Anderen als Ursache der bleibenden Gehörsstörungen angeschuldigt werden. Immerhin sind gegen die Annahme, dass diese beschriebenen hyperämischen Zustände und die gefundenen Hämorrhagien auf eine specifische Chininwirkung zurückzufuhren sind, verschiedene Bedenken zu erheben.

1) Kirchner, Extravasate im Labyrinth durch Chinin- und Salicylwirkung. Monatsschr. f. Ohrenheilk. $1883 \mathrm{Nr} 5$.

2) Gherardo Ferreri, Le emorragie dell' orrecchio interno. Archivio Italiano di otologia etc. Bd. 12 H. 4. 1902. 
Schon Jacoby ${ }^{1}$ ) machte bei der Besprechung der Kirchnerschen Arbeit darauf aufmerksam, dass es zweifelhaft bliebe, ob die sehr ausgesprochenen Suffocationserscheinungen, unter denen die mit Chinin vergifteten Thiere zu Grunde gehen, oder das Chinin selbst als Ursache der gefundenen Hyperämie bezw. Blutungen anzusprechen seien. Aebnliche Bedenken erhoben $\mathrm{MoOs}^{2}$ ) und Gradenigo ${ }^{3}$ ). Nun hat zwar Grunert ${ }^{4}$ ) den Versuch gemacht, diese Bedenken za entkräften. Er tödtete eine Taube und eine Maus durch Strangulation und untersuchte die Schläfenbeine dieser beiden Thiere mikroskopiseh in Serienschnitten. Er fand ebenso wie Kirchner Hyperämie und reichliche Bluterguisse in der Paukenhóhle; ja sogar in den perilymphatischen Raumen des Labyrinthes und in der Scala tympani konnte er ausgedehnte Blutextravasate nachweisen. Da es ihm indessen nicht gelungen war, experimentell durch Suffocation Blutergússe auch in den endolymphatischen 'Räumen des Gehöl'organes zu erzeugen, wie sie Kirchner erzielt hatte, hält er es trotzdem fur wahrscheinlich, dass die von K i c chuer nachgewiesenen Blutungen in den Ductus cochlearis hinein einer specifischen Giftwirkung zuzuschreiben seien.

Wenn man aber bedenkt, dass Grunert nur zwei Thiere untersuchte, Kirchner aber diese Blutungen in dịe endolymphatischen Räume offenbar nur vereinzelt gefunden hat, so kann man die Grunert'schèn Béfunde mit ebensoviel Recht gegen als fú $r$ die Kirchner'schen Schlussfolgerungen verwerthen.

Das Gleiche gilt von den Untersuchungen Ferreri's. Scheint ihr erster Theil eine Bestätigung der Kirchner'schen Befunde zu geben, so gibt der zweite Theil einen Beweis für die Berechtigung des oben erwähnten Einwảndes, denn in ihm weist er nach, dass beim Suffocationstod genau die gleichen Veränderungen im Labyrinth und Akustikus zu finden sind wie bei Chinintoxication. Dass er die Blutungen im Labyrinth nach Chinintoxication so auffallend constant fand, würde dann damit zu erklären sein, dass gerade bei Katzen die Vergiftungserscheinungen sehr schwere und die Respirationsstörungen während der Agone sehr ausgesprochen sind.

1) Jacoby, Archiv f. Ohrenheilk. Bd. 18 S. 305.

2) Moos, a. a. 0 .

3) Gradenigo, a. a. 0.

4) Grunert, Zur Kritik der thierexperimentellen Ergebnisse Ki r clı ner's u. s. w. Archiv f. Ohrenheilk. Bd. 45 s. $161 \mathrm{ff}$. 
Der zweite Einwand stutzt sich auf den Widerspruch zwischen den Sektionsbefunden Kirchner's in der Paukenhöhle und den otoskopischen Befunden, die bei den experimentell mit Chinin vergifteten Personen erhoben wurden. Eine Blutung ist hierbei niemals beobachtet worden, und die Beobachtung einer Injection der Hammergriffgefässe durch Roosa hat von anderer Seite keine Bestätigung gefunden.

Auch mit den Veränderungen an der Retina, die der Hörstörung analoge Störungen des Sehvermoggens bedingen, sind die Kirchnerschen Befunde nicht vereinbar. Dass hier regelmässig eine ausgesprochene Anamie der Netzhaut vorhanden ist, ist zweifellos. Blutungen in der Netzhaut selbst sind, meines Wissens klinisch niemals gefunden worden. Auch hierauf hin angestellte experimentelle Untersuchungen sind negativ ausgefallen; (Ulrich ${ }^{1}$ )

Uhtboff ${ }^{2}$ ) schreibt: „Acute Entzúndúngsprocesse oder Blutergiusse in den Opticusstämmen oder stärkere Blutungen resp. seróse Ergủsse in die Sehnervenscheidenräume mit Strangulation des Sehnerven dürften fur die Exklärung der. Chininamblyopie nicht in Betracht kommen." Vorkommen von Blutungen in der Retina finde ich nicht erwảhnt.

Schliesslich erscheint auch vom allgemeinen pharmakologischen Standpunkte aus die Annahme, dass das Chinin durch specifische Giftwirkung im Stande sei, Blutungen hervorzurufen, nicht erklärlich, da sonst eine blutungerzeugende Wirkung des Chinins bei Thieren niemals zu beobachten ist.

Wo beim Menschen derartige Störungen beschrieben wurden, handelte es sich in der Regel um kranke oder in ihrem allgemeinen Ernährungszustand stark beeinträchtigte Individuen.

Während also auf Grund der klinischen Beobachtungen und experimentellen Untersuchungen am Menschen als feststehend angesehen werden muss, dass der Sitz der bèi Chininintoxicationen auftretenden Börstörungen im Labyrinth gelegen ist, ist über die Art der zu Grunde liegenden Veränderungen noch keine Klarheit geschaffen. Vielmehr stehen sich hier zwei Anschaungen gegenuber,

1) Ulrich, Ueber Netzhautblutungen bei Anamie etc. v. Graefe' Archiv f. Ophthalm. Bd. 38 H. 2 S. 1.

2) Uhth off, Ueber die Augenstorungen bei Vergiftungen. Gra efeSaemisch, Handbuch d. Augenheilk. 2. Aufl. Bd. 11 Cåp. 22 Th. 2. 
von denen die eine nach Analogie mit den der Sehstörung zu Grunde liegeǹden Circulationsstörungen eine $\mathrm{Anämi}$ e der häutigen Theile des Labyrinthes annimmt, während die andere auf Grund der Kirchner'schen Untersuchungen eine Hyperämie, die in besonders sehweren Fällen sogar zu Blutaustritten und entzündlichen Veränderungen fuhren könne, als Ursache fur die Hörstorung anruft.

Eine Nachprifung der Kirchner'schen Untersuchung erschien demnach als wunschenswerth; eine solche habe ich, einer Anregung neines Chefs, Herrn Prof. Kummel, folgend, unternommen.

Ich habe eine grosse Anzahl von Thieren (Kaninchen, Katzen, Meerschweinchen) in ähnlicher Weise, wie dies Kirchner angibt, sei es durch Application der Schlundsonde, sei es durch subcutane Injection mit Chininum muriat., vergiftet. Fin Theil erhielt grosse, oft sehon nach erstmaliger Application, sonst in der Regel nach zweibis dreimaliger Wiederholung tödtliche Dosen, ein anderer Theil ca. acht Tage hindurch kleinere Dosen, die bei einmaliger Application niemals tödtlich waren und kaum merkliche Vergiftungserseheinungen hervorriefen. Bei diesen Thieren erfolgte der Tod oft ganz unerwartet, meist sehr kurze Zeit nach Application der letzten Dosis. Die Empfindlichkeit der einzelnen Thiere gegenuber dem Gifte schwankt in weiten Grenzen; während z. B. im Allgemeinen $1,0 \mathrm{~g}$ Chinin. muriat. per Schlundsonde von Kaninchen meist noch uberstanden wurde, trat einmal schon nach 0,5 g der Exitus ein. Bei subcutaner Injection war die todtliche I)osis durchschnittlich etwas geringer. Zur Verabreichung durch die Schlundsonde wurde das Gift mit Gummiarabicum verrieben. Nach der subcutanen Injection kam es bei einigen Meerschweinchen, im Allgemeinen aber sehr selten, an der Injectionsstelle zur Bildung eines kleinen Abscesses, falls die Tiere noch einige Tage am Leben blieben.

Die Vergiftungserscheinungen, unter denen die Thiere zu Grunde gingen, waren die gleichen, wie sie Kirchner beschreibt. Die ersten Erscheinungen beginnen in der Regel $1 / 4$ bis $1 / 2$ Stunde nach Application des Giftes damit, dass das Thier anfangt, taumelnde Bewegungen zu machen und beim Versuch zu laufen, hin und her schwaukt. Dann macht sich in der Regel zuerst die lähmende Wirkung an den vorderen Extremitäten bemerkbar, so dass das Thier jetzt in ganz charakteristischer Haltung bei weit gespreizten Vorderbeinen mit dem Brustkorb aufliegt. Die Pupillen zeigen eine deutliche Erweiterung, und die Cornealreflexe werden schwächer. 
Allmählich sieht man auch die hinteren Extremitäten versagen. Jetzt folgen eine Zeit hindurch, "anfangs noch mit Erfolg, dann vergeblich ausgefuhrte Aufrichtungsversuche, bis das Thier schliesslich auf der Seite liegen bleibt mit muhsamer, vertiefter und stark beschleunigter Respiration. Die Pupillen sind jetzt maximal erweitert, Cornealreflexe und sammtliche andere Reflexe gänzlich erloschen. Bei Kaninehen erfolgt in der Regel sehr bald, nach kurzem Opisthotonus und Streckkrampfen der Extremitäten und nach einigen schnappenden Athmungsbewe@ungen der Exitus. Bei den Meerschweinchen und Katzen dagegen können, falls die verabreichte Dosis die eben tödtliche nicht zu sehr übertrifft, Opisthotonus und Streckkrämpfe Stunden lang anhalten, in kurzen, regelmässigen Intervallen wiederkehrend, bis sie dann allmählich aufhören und der Tod in gleicher Weise wie bei den Kaninchen erfolgt. Bei Katzen stellt sich ausserdem schon kurz uach der Application des Giftes unter kläglichem Geschrei eine sehr starke Salivation und auch Erbrechen ein. Die lángere Zeit hindurch mit kleinen Dosen gefütterten Thiere gehen unter ähnlichen, aber meist längst nicht so intensiven Erscheinungen zu Grunde. Einige Thiere erlagen nach verhältnissmässig langer Zeit noch nachträglich der Vergiftung, nachdem sie sich anscheinend schon wieder etwas erholt hatten; sie wurden morgens todt aufgefunden.

Fine Hörstörung bei den vergifteten Thieren festzustellen, stiess auf sehr grosse Schwierigkeiten. Dass bei den mit grossen Dosen vergifteten Thieren auf der Höhe der Intoxication mit Erlöschen der Reflexerregbarkeit auch die Reaction auf akustische Reize schwand, heweist nicht das Vorhandensein einer auf Alteration des Gehörorganes zurückzufuhrenden Schwerhörigkeit bezw. Taubheit. Bei den mit kleinen Dosen während langer Zeit vergifteten Thieren hatte ich zwar zuweilen, namentlich bei Meerschweinchen, den Eindruck, als ob hie Reaction auf akustische Reize (schrille Pfffe, Zusammensehlagen zweier Schussel u. dgl.) nicht mehr so prompt erfolgte wie zuvor. Doch war sie niemals voillig erloschen, so dass ich hierauf keinen grossen Werth legen möchte, da Täuschungen hierbei nicht ausgeschlossen sind. Einmal glaubte ich bei einer Katze eine Ueberempfindlichkeit gegen akustische Reize feststellen zu können.

Die Untersuchung der möglichst bald nach dem Tode herausgenommenen Schläfenbeine erfolgte mikroskopisch nach Anlegung von Serienschnitten. 
Sie wurden in $\mathrm{M}$ ü1ler-Formalinlösung fixirt, in Salpetersaure-Formalinlósung entkalkt, ausgewaschen, in steigendem Alkohol gehartet und in Celloidin eingebettet. Die Farbung der angefertsgten Serienschnitte erfolgte mit Hamatoxilin-Eosin, und auch nach van Gieson.

Schon die Untersuchung der Schläfenbeine des ersten Versuchsthieres machte mich auf einen Umstand aufmerksam, der zu groben Táuschungen Veranlassung geben kann und den Kirehner bei der Beurtheilung seiner Befunde nicht berucksichtigt zu haben scheint.

Der Versuch war folgender:

Kaninchen, $1700 \mathrm{~g}$ schwer. 21. October 1901. 0,8 g Chinin. mur. per Schlurdsonde, zeigt hiernach massig schwere Vergiftungserscheinungen, erholt sich nach ca. 1 Stunde wieder.

22. October 1901. 1,0 g Chinin. mur. per Schlundsonde. Exitus nach 40 Minuten unter'schweren Vergiftungserscheinungen, wie sie oben beschrieben. Eine auf der Hohe der Vergiftungserscheinungen vorgenommene Besichtıgung des Trommelfelles konnte an ihm keine Injection nachweisen.

Allgemeinsection: Kleme Petechien am Endokard und der Pleura, sonst nirgends Blutungen.

Schlafenbeine, beiderseits gleicher Befund: Zahlreiche frische kleine Blutungen in der Paukenhohlenschleimhant, erweiterte Gefasse, daneben aufgelagertes Blut an der Stelle, wo die Paukenhohle vor der Fixirung eroffnet war. Im Labyrinth an mehreren Stellen aufgelagertes Blut so: in der Scala tympani, am Boden des Vestibulums, theilweise anch in den endolymphatischen Raumen, die am Boden des Vestibulums sich eroffinet erwiesen. Unzweifelhaft im Gewebe selbst liegende kleine Blutungen, analog denen in der Paukenhoblenschleimliaut, sind im Labyrinth nicht aufzufinden.

In diesem Versuch hatte ich, ausgehend von dem Gedanken, eine bessere Fixirung zu ermöglichen, bevor ich die Schläfenbeine in die Fixirungsflussigkeit einlegte, durch Wegnahme eines Theiles der Promontorialwand das Labyrinth eróffuet; der Versuch zeigt deutlich, dass es in diesem Falle ausserordentlich schwer, geradezu unmöglich war, mit Sicherheit zu entscheiden, ob die gefundenen Blutanhäufungen ihre Entstehung Blutaustritten verdanken, die erst wahrend der Eroffnung der Labyrinthkapsel, bei der eine Zerreissung der häutigen Wand des Labyrinthes nicht vermeidbar ist, zu Stande gekommen sind oder ob es sich thatsächlich um intra vitam entstandene Blutung handele. Denn ein wesentlicher Untersehied der post mortem entstandenen Blutansammlungen von einer intravitalen Blutung in der Anordnung der Blutkörperchen zu einander (Geldrollenbildung u. dgl.), sowie in dem Aussehen der einzelnen Blutkörperchen selbst (Degenerationsformen) ist kaum zu erwarten, wenn man bedenkt, dass die 
Beitrage zur Kenntniss der Wirkung des Chinins auf das Gehororgan. 223

Schläfenbeine in der Regel lebenswarm, unmittelbar post mortem fixirt wurden. Der wichtigste Punkt scheint mir der zu sein, dass wir fur die Annahme einer intravitalen Blutung die Lagerung der Blutkorperchen, wenigstens theilweise, im Gewebe selbst verlangen müssen. Das war auch bei den Blutungen in der Paukenhöhlenschleimhaut der Fall; dagegen ist mir furr reine Auflagerungen bei völliger Intactheit des unterliegenden Gewebes, sei es nun der Paukenhöhlenschleimhaut oder des häutigen Labyrinths, die Annahme einer post mortalen Entstehung wahrscheinlicher.

So kann man sich denn auch beim Anblick der von Kirchner ${ }^{1}$ ) gegebenen Abbildungen des Eindrucks nicht erwehren, dass es sich hier sehr wohl um reine Blutauflagerungen handeln könne. Auch spricht der Nachweis, dass das Blut aus einem grosseren Gefässe stammt, entschieden gegen eine intra vitam und fur eine post mortal entstandene Blutansammlung, denn im ersteren Falle wäre es viel wahrscheinlicher, dass es sich um capilläre Blutungen oder mindèstens um Blutungen aus kleinen, zartwandigen Gefässen handeln würde. Ich habe daher in den folgenden Versuchsprotokollen von einer Erwahnung unzweifelhaft aufgelagerter Blutungen auf intacter Paukenhöhlenschleimhaut, wie sie sich sehr häufig in der Umgebung der Stelle finden, an der diese, theils unabsichtlich, theils auch absichtlich, eröffnet worden war, abgesehen. Um aber das nachträgliche Hineingerathen von Blut in das Labyrinth zu vermeiden, babe ich von einer der Fixirung vorhergehenden Eröffuung desselben abgestanden. Die weiteren Versuche fielen nun folgendermassen aus:

\section{Durch ein- bezw. mehrmalige Application grosser Chinindosen getödtete Thiere.}

1. Kaninchen, $1500 \mathrm{~g}$ schwer. 21. October 1901. $0,8 \mathrm{~g}$ Chinin. mur per Schlundsonde: bald leichte Vergiftungserscheinungen. - 22. October 1901. $1,0 \mathrm{~g}$ Chinın. mur. per Schlundsonde: massig schwere Vergiftungserscheinungen, erholt sich indessen wieder. Keine Injection am Trommelfell. - 23. October 1901. Erscheint nach nicht wieder vollig munter. 0,8 g Chinin. mur. per Schlundsonde; schwere Vergiftungserscheinungen, denen das Thier schon nach $1 / 4$ Stunde erliegt.

Allgemeinsection: Kleine Petechien an Pleura und Endokard, sonst nirgends Blutungen.

Schlafenbein: Beiderseits kleine Hamorrhagien der Paukènhóhlenschleimhaut; erweiterte Gefásse im linkèn Schlafenbein, ausserdem ziemlich reichliche

1) Kraner, a. a 0. 
Blutansammlung am Boden des Vestibulums, hereinreichend bis in die Scala vestibuli der Schnecke. Diese Blutansammlung ist frei in den perilymphathischen Rảumen gelegen, wàhrend das hautige Labyrinth vollig intact erschemt. Bei ihrer Verfolgung durch die einzelnen Serienschnitte hindurch zeigt sich, dass sie an der Einmündungsstelle einer gröss eren Knochenvene ihren Anfang nimmt. Rechtes Labyrinth intact.

2. Katze, $2100 \mathrm{~g}$ schwer. 23. October 1901. 1,0 g Chinin. mur. per Schlundsonde, ca. 2 Stunden lang anhaltende, schwere Vergiftungserscheinungen, von denen das Thier sich indessen allmahlich wieder erholt. Trommelfell zeigt keine Injection. Einige Stunden nachher deutlich gesteigerte Reflexerregbarkeit. 24. October 1901. Das Thier erscheint noch nicht wieder vollig erholt, hat schlecht gefressen. 0,5 g Chinin. mur. per Schlundsonde. Schon nach 20 Minuten Einsetzen schwerer Vergiftungserscheinungen, nach 50 Minnten Einsetzen intensiver, an Strychninintoxication erinnernder Krampfe, die eine ganze Stunde anhalten, dann ertolgt Exitus in der beschriebenen Weise. Injection am Trommelfell war auf der Höhe der Vergiftung nicht nachzuweisen.

Allgemeinsection: Petechien an Pleura und Endokard, sonst nirgends Blutungen.

Schläfenbeine: Beiderseits, aber sehr vereinzelt, kleine Hamorrhagien der Paukenhohlenschleimhaut.

Linkes Labyrinth: Neben stark gefullten Gefassen in der Paukenbohlenschleimhaut stark gefulltes Gefass im Vestibulum; anscheinend eine Blutung im Akustikusstamme. Bei Verfolgung der Serienschnitte zeigt sich, dass das Blut einem an der Durchtrennungsstelle des Nerv. acusticus mit durchschnittenen grósseren Gefass entstammt.

Rechtes Labyrinth: An der Einmundungsstelle des Aquaeductus cochleae geringe Anhaufung von vollig frei in den perilymphathischen Raumen liegenden rothen Blutkorperchen.

3. Meerschweinchen, $380 \mathrm{~g}$ schwer. 15. November 1901. $0,1 \mathrm{~g}$ Chinin. mur. subcutan. Schon nach 10 Minuten Einsetzen der Vergiftungserscheinungen, die sich nach 30 Minuten bis zum Einsetzen von Krampfen wie bei dem zweiten Versuchsthier steigern, ca. 40 Minuten anhaltend. Dann erfolgt sehr bald der Exitus.

Allgemeinsection: Petechien in Pleura und Endokard, sonst nirgends Blutungen.

Schláfenbein: Beiderseits kleine Hamorrhagien in der Paukenhöhlenschleimhaut und Hyperamie derselben.

Links: Anscheinend eine Blutung im Akustikus, doch lasst sich bei Verfolgung der Serien feststellen, dass das Blut aus einem gróss eren, am centralen Stumpf des Akustikusstammes durchschnittenen Gefasse stammt. Labyrinth beiderseits intact.

4. Katze, $2500 \mathrm{~g}$ schwer. 28. November 1901. 0,5 g Chinin. mur. per Schlundsonde; leichte Vergiftungserscheinungen. - 5. December $1901 \quad 0,8 \mathrm{~g}$ Chinin. mur. per Schlundsonde. Massig schwere Vergiftungserscheinungen, erholt sich wieder. Trommelfell ohne 1.jjection. - 6. December 1901. Erscheint noch sehr apathisch, 
Beitrage zur Kenutnıs der Wrkung des Chinms auf das Gehororgan. 225

reagirt auf laute akustische Reize sehr trage. -13 . December 1901. Erscheint wieder vollig erholt und reagirt gut. $1,0 \mathrm{~g}$ Chinm. mur. per Schlundsonde; noch etwas intensivere Wirkung wie am 5 December; erholt sich auch diesmal wieder. 18. December 1901. Chinin. mur. $0,5 \mathrm{~g}$ subcutan. Hiernach Einsetzen schwerer Vergiftungserscheinungen, die indessen an Intensitat wesentlich geringer sind als die des zweiten Versuchsthieres und bald in ein Stadium volliger Somnolenz mit volliger Reflexlosigkeit ubergehen. Nach ca. 1 Stunde tritt unter allmahlich schwacher werdender Respiration der Exitus ein.

Allgemeinsection: Keine Blutungen, auch nicht in Pleura und Endokard, auch keme alteren Heerde 1

Schlafenbeine: Nur linkerseits einige kleine Hamorrhagien der Paukenhohlenschleimhaut. Labyrinth beiderseits intact.

5. Kaninchen, $1350 \mathrm{~g}$ schwer. 9. Januar 1902 . 1,0 $\mathrm{g}$ Chinin. mur. subcutan. Ziemlich schwere Vergiftungserscheinungen, von denen es sich am Abend anscheinend schon wieder erholt hatte. - Am 10. Januar 1902 morgens todt aufgefunden.

Allge meinsection: Einige Petechien an der Pleura, sonst keine Blutungen.

Schlafenbein: Beiderseits chronische Mittelohreiterung, rechts mit Betheiligung des Labyrinthes. Nirgends Blutungen, weder im Mittelohr noch im Labyrinth.

6. Meerschweinchen, $410 \mathrm{~g}$ schwer. 15. Januar 1902, 0,01 g Chinin. mur. subcutan. Sehr schwere Vergiftungserscheinungen mit Krampfen, von denen es sich am Abend schon wieder etwas erholt hatte; trotzdem Exitus wahrend der Nacht.

Allgemeinsection: Petechien in Pleura und Endokard, sonst nirgends Blutungen.

Schlafenbeine: Beiderseits kleine Hamorrhagien der Paukenhohlenschleimhant und Hyperảmie derselben. Beiderseits intactes Labyrinth; linkerseits sehr stark gefullte Gefasse in der Schnecke.

7. Meerschweinchen, $460 \mathrm{~g}$ schwer. 10. Februar 1902. $0,1 \mathrm{~g}$ Chinin. mur. subcutan Sehr schwere Vergiftungserscheinungen. Verhalten wie beim sechsten Versuchsthier.

Allgemeinsection: Kleine Petechien in Pleura und Endokard, sonst nirgends Blutungen.

Schlafenbeine: Beiderseits kleine und nur recht vereinzelte Hamorrhagien und Hyperamie der Paukenhỏhlenschleimhaut. Labyrinthe intact.

8. Kaninchen, $1100 \mathrm{~g}$ schwer. 6. November 1901. 0,5 g Chinin. mur. subcutan Keine auffalligen Vergiftungserscheinungen. - 7 November 1901. Morgens todt aufgefunden.

Allgemeinsection: Pneumonische Infiltrate beider Oberlappen. Petechien in Pleura und Endokard, sonst nirgends Blutungen.

Schlafenbeıne: Nirgends Blutungen oder auffallige Hyperamie. Labyrinthe beiderseits intact.

E. Pfituger, Archiv für Physiologie. Bd. 95. 


\section{Durch längere Zeit hindurch täglich verabreichte, kleinere Dosen vergiftete 'Thiere.}

1. Kaninchen, $1200 \mathrm{~g}$ schwer. 7. November 1901 . $0,5 \mathrm{~g}$ Chinin. mur. per Schlundsonde; leichte Vergiftungserscheinungen, erholt sich indessen wieder. 8. November und 9. November 1901. 0,5 g Chinin. mur.; etwas dentlichere Vergiftungserscheinungen, von denen es sich wieder erholt. Keine Injection am Trommelfell. - 10 November 1901. 0,5 g Chinin mur. Verhalten wie am 9. November 1901. - 11. November 1901. 0,5 g Chinin. mur Kurz danach erfolgte unter verhaltnissmassig schweren Vergiftungserscheinungen schnell der Exitus.

Allgemeinsection: Reichliche Petechien in der Pleura.

Schlafenbeine: Beiderseits ziemlich reichliche kleine Hamorrhagien in der Paukenhohlenschleimhaut, daneben stark gefullte Gefasse, auch im Labyrinth.

Rechts: In den perilymphathischen Raumen der Scala vestıbulı der letzten Schneckenwindung geringe Anbaufung vollig freiliegender rother Blutkorperchen, ohne Zerreissung der Wandung des Ductus cochlearis.

Links intactes Labyrinth.

2. Kaninchen, $2150 \mathrm{~g}$ schwer. - 25 October 1901. 0,5 g Chinin. mur. per Schlundsonde; nur leichte Vergiftungserscheinungen. Trommelfelle ohne Injection. - 26 October 1901. 0,5 g Chinin. mur. - 28. October 1901. 0,5 g Chinin. mur. - 29. October. 1901. 0,5 g Chinin. mur. - 31. October 1901. 0,5 g Chinin. mur. Wirft vier nicht ausgetragene, aber noch lebende Junge. -1 . November 1901. $0,5 \mathrm{~g}$ Chinin. mur., ebenso am 2. und 3 . November. Gewicht $1 \mathrm{~kg} 530 \mathrm{~g}$. 5. November 1901. 0,5 g Chinı. mur., erschent jetzt sehr ruhig, haart stark, frisst schlecht. - 6. November 1901. Morgens todt aufgefurden.

Allgemeinsection: Hypostasen in den Unterlappen der Lunge. Kleine Hamorrhagien in Pleura und Endokard Sonst nirgends Blutungen.

Schlafenbeıne: Beiderseits nur einige stark gefullt erscheinende Gefasse. Nirgends Blutungen. Labyrinth beiderseits intact.

3 Meerschweinschen, $450 \mathrm{~g}$ schwer. 7. November 1901. $0,5 \mathrm{~g}$ Chinn. mur. subcutan. Hiernach ausgesprochene Vergiftungserscheinungen; erholt sich indessen wieder. - 9. November 1901. 0,05 g Chinin. mur. subcutan, ebenso am 11. und 12. Novernber, gefolgt von sehr leichten Vergiftungserscheinungen. 14. b1s 16. November taghich $0,05 \mathrm{~g}$ Chinın. mur.; erscheint nicht mehr vollig so munter wie vorher, verhalt sich ruhiger, frisst schlechter. - 18. November 1901. 0,05 g Chinin. mur. - 19. November 1901. 0,1 g Chinin. mur.; nach 20 Minuten schwere Vergiftungserschemungen, langere Zeit anlaltend, doch scheint es sich am Abend schon wieder etwas erholt zu haben. Morgens todt aufgefunden.

Allgemeinsection: Vereinzelte Petechien in Pleura und Endokard. Sonst nirgends Blutungen.

Schlafenbeine: Beiderseits veremzelte kleme Blutungen der Paukenhohlenschleimhaut, vereinzelt auch stark gefullte Gefasse. Labyrnnthe vollig intact.

4. Meerschweinchen, $390 \mathrm{~g}$ schwer. 28. November 1901. $0,05 \mathrm{~g}$ Chnin. mur. subcutan; nur leichte Verglftungserscheinungen. - 29. November 1901 bis 
Beitrage zur Kenntniss der Wirkung des Chinins auf das Gehörorgan. 227

1. December je $0,05 \mathrm{~g}$ Chinin. mur. subcutan; sitzt auffallend ruhig, frisst schlechter. - 6. December 1901. 0,05 g Chinin. mur.; leichte Vergiftungserscheinungen. - 7. December 1901. Morgens todt aufgefunden.

Allgemeinsection: Keine Petechien in Pleura und Endokard; beginnende eitrige Peritonitis, nirgends Blutungen.

Schlafenbeine: Nirgends Blutungen in der Paukenhohlenschleimhaut L'abyrinthe intact.

$\therefore \quad$ Ich hebe hervor, dass die angeführten Versuchsprotokolle in wesentlich abgekürzter Form wiedergegeben worden sind, und dass nur die wichtigsten Punkte aus den einzelnen Versuchsreihen erwähnt wurden. Eine etwas stärkere Füllung der einzelnen Gefässe der'Paukenhöhle, und auch des Labyrinthes, als wir dies bei z. B. wurch Chloroform getödteten Kaninchen zu sehen pflegen, ist in fast sảmmtlichen Versuchen aufgefallen. Sie war im Allgemeinen um so ausgesprochener, unter je heftigeren Vergiftungserscheinungen (Krämpfe, Dyspnoë u. s. w.) die Thiere zu Grunde gegangen waren.

Wenn wir das Resultat dieser Versuchsreihen zusanmenfassen sollen, so ist in erster Linie zu betonen, dass im Ganzen nur drei Mal (I 1 u. 2 und II 1) Blutansammlung im Labyrinth beobachtet wurde. Hierbei handelte es sich lediglich um frei in den perilymphatischen Rảumen liegendes Blut, wảhrend die end olymphatischen Räume sowie die Wandungen des hautigen Labyrinthes sich in sảmmtlichen Versuchen als völlig unverändert erwiesen. Hervorhebenswerth erscheint noch, dass sich bei Verfolgung der Blutansammlungen durch die einzelnen Serienschnitte mit grosser Wahrscheinlichkeit feststellen liess, dass das Blut aus einer in die perilymphatischen Raume mundenden g r ö s s e r en Knochenvene bezw. der Vene des Aquaeductus cochleae entstammte. Kleine Blutungen in der Paukenhöhlenschleimhaut selbst sind dagegen ein fast regelmássiges Vorkommniss bei den der Chininintoxication erlegenen Thieren, ebenso eine mehr oder weniger deutlich ausgesprochene stärkere Füllung der Gefässe in Paukenhöhle und Labyrinth. Das Gleiche gilt für das Vorkommen von Petechien in Pleura und Endokard, während Blutungen in den ubrigen Organen niemals aufgefunden wurden. Die Blutungen in der Paukenhỏhlenschleimhaut sowohl als auch die in der Pleura und im Endokard finden sich am reichlichsten bei denjenigen Thieren, welche unter den heftigsten Vergiftungserscheinungen (Krämpfen, starker Dyspnoë) zu Grunde gingen (z B.I1, 2 u. 3 u. II 1), wàhrend sie bei solchen, die mehr allmählich dem der Vergiftung 
folgenden allgemeinen Schwächezustand nach vorheriger anscheinender Erholung erlagen, zuweilen gånzlich vermisst wurden (z. B. I 8, II 4).

Bluterguisse im Akustikusstamm, wie sie von Ferreri beschrieben wurden, habe ich bei zwei Versuchsthieren (I 2 u. 3) aufgefunden. Auch bei diesen liess sich bei Verfolgung des Blutes durch die einzelnen Serienschnitte seine Herkunft aus einem offenbar mit dem Akustikus bei der Herausnahme des Gehirns durcbschnittenen grösseren Gefässe feststellen. Veränderungen an den Gefässwänden, Proliferation des Endothels oder Aehnliches babe ich niemals auffinden können.

Bei der Besichtigung des Trommelfelles im Höhestadium der Intoxication habe ich niemals auffallende Injection oder gar kleine Petechien daran auffinden kỏnnen. Die Untersuchung lässt sich bei Kaninchen und Katzen, wenn man vorher fưr Reinigung des Gehörganges Sorge getragen hat, mit einem langen engen Ohrtrichter in der Regel ganz gut durchfuhren.

Ich glaube demnach auf Grund dieser Untersuchungen zu folgenden Schlussfolgerungen berechtigt zu sein: Das Auftreten von Blutungen im Labyrinth bei den an Chininintoxication zu Grunde gegangenen Thieren ist, falls die oben erwähnten Blutanhäufungen überhaupt als intravital entstandene Blutungen aufzufassen sind, ein zum Mindesten seltenes Vorkommniss. Das Gleiche gilt von den von Ferreri erwähnten Blutungen in den Akustikusstamm. Kleine Blutergüsse in der Paukenhöhlenschleimhaut sowie stärkere Fullung der Gefässe in Paukenhöhle und Labyrinth sind, ebenso wie die Petechien in Pleura und Endokard, bei den unter heftigen Krämpfen und äusserst forcirten Athembewegungen $\mathrm{zu}$ Grunde gegangenen Thieren regelmässig aufzufinden. Dagegen können sie fehlen bei den Thieren, bei denen der Verlauf der Vergiftung weniger stürmisch war. Gerade dieser letztere Umstand legt den Gedanken nahe, die gefundenen Blutungen ebenso wie die starke Erweiterung der Gefässe nicht als auf specifischer Giftwirkung beruhend, sondern als secundäre aufzufassen. Als eigentliche Ursache wurden dann die schweren Suffocationserseheinungen, unter denen die vergifteten Thiere zu Grunde zu gehen pflegen, und die durch sie bedingte intensive venóse Stauung anzunehmen sein. Für diese Annahme sprechen ferner der negative Ausfall der auf der Höhe der Intoxication vorgenommenen Trommelfelluntersuchungen und die Versuche 
Grunert's, der die gleichen Veränderungen - starke Füllung der Gefässe in Paukenhöhle und Labyrinth, Blutungen in der Paukenhöhlenschleimhaut und Blutansammlungen in den p erilymphatischen Räumen - bei erdrosselten Thieren nachweisen konnte. Einen. weiteren Anhaltspunkt fur diese Annahme bieten die Untersuchungen Ferreri's, der, wie schon erwähnt, bei den dem Erstickungstode erlegenen Thieren vollstandig analoge Befunde erhob wie bei denen, die an einer Chininintoxication zu Grunde gegangen waren.

Bezugglich der Blutungen im Akustikusstamm ist ferner die Frage zu erwägen, ob sie überhaupt als intravitale Blutungen aufzufassen sind oder auf Tänschungen beruhen, dadurch entstanden, dass bei der Durchtrennung des Akustikusstammes, während der Herausnahme des Gehirns, sich Blut aus einem hierbei mit durchtrennten grösseren Gefảsse nachträglich in den Nervenstamm ergossen hat. Ebenso könnten die Blutansammlungen in den perilymphatischen Räumen durch Hereinpressen von Blut aus einer prall gefüllten Knochenvene bezw. der Vene des Aquaeductus cochleae während der Herausnahme der Schlafenbeine erklärt worden.

Um auch hieruber völlige Klarheit zu erhalten, habe ich mich bemuht, in einer weiteren Versuchsreihe sämmtliche Fehlerquellen, die in dieser Beziehung Täuschungen hervorrufen konnten, auszuschalten. $\mathrm{Zu}$ diesen gehört z. B. auch die Application des Giftes mit Schlundsonde. Sie ist nur nach Aufspannen des Thieres auf einen Thierhalter möglich, wobei der Hals des Thieres von zwei eisernen Klammern umfasst wird. Fängt das Thier an sich zu sträuben und Abwehrbewegungen zu machen, so stösst es sehr leicht gerade mit dem Boden der Paukenhöhle gegen die beiden eisernen Klammern, und ich habe thatsächlich bei einer Katze lediglich nach dem Aufspannen, obne dass ich ihr Chinin applicirt batte, reichliche kleine Hämorrhagien in der Paukenhöhle feststellen können. Auch muss, falls die Thiere in das Krampfstadium verfallen, selbstverständlich Aufschlagen des Kopfes gegen den Boden oder die Wandung des Käfigs vermieden werden. Ich habe mich daher in dieser neuen Versuchsreihe stets der subcutanen Application des Giftes bedient. Ich schlug folgendes Verfahren ein: Die theils durch eine einmalige sicher tödtliche Dosis, theils durch längere Zeit hindurch verabreichte kleinen Dosen vergifteten Thiere wurden, kurz bevor der Exitus zu erwarten war, einmal durch Combination von Verblutung mit 
Durchspülung des Gefässsystems (mit isotonischer Kochsalzlösung), in den übrigen Fällen einfach durch Entblutung nach Durchtrennung beider Karotiden und Jugularvenen getödtet. Hierdurch war die Möglichkeit des Zustandekommens einer stärkeren venösen Stauung im Gefássgebiet der Paukenköhle und des Labyrinths während der Agone ausgeschlossen. Um ferner bei der Herausnahme des Gehirns das nachträgliche Eindringen von Blut in den Akustikusstamm nach dessen Durchtrennung, und bei der Herausnahme der Schläfenbeine die Móglichkeit des Hereinpressens von Blut in die perilymphatischen Räume auszuschliessen, wurden zunächst die ganzen Kỏpfe der Thiere nach vorsichtiger Eröffnung der Schädelhöhle von Foramen magnum aus in die Fixirungsflussigkeit übertragen und erst mehrere Stunden später, als also das Vorhandensein noch flussigen Blutes innerhalb der Gefässe ausgeschlossen war, die Schläfenbeine selbst herauspräparirt. Ihre weitere Behandlung glich völlig der in den vorhergehenden Versuchsreihen angewandten. Die einzelnen Versuche gestalteten sich, ebenfalls in abgekurzter Form wiedergegeben, folgendermaassen:

\section{Durch einmalige Application einer grossen Dosis getödtete Thiere.}

1. Kaninchen, $1300 \mathrm{~g}$ schwer. Erhalt $1,0 \mathrm{~g}$ Chinin. mur.; nach $1 / 2$ Stunde schon schwere Vergiftungserscheinungen, nach 50 Minuten völlige Lahmung, Reflexlosigkeit, beschleunigte keuchende Athmung, so dass der Exitus nahe bevorstand. Todtung durch Spulung.

Allgemeinsection: Vereinzelte kleine Petechien in der Pleura, sonst nirgends Blutungen.

Schlafenbeine: Beiderseits weder im Mittelohr noch im Labyrinth oder Akustikusstamm Blutergusse.

2. Kaninchen, $1500 \mathrm{~g}$ schwer. Erhalt $1,0 \mathrm{~g}$ Chinin. mur. Beginn der Vergiftungserscheinungen nach $1 / 2$ Stunde. Nach zwei Stunden völlige Erschöpfung, so dass der Exitus bevorstand. Todtung durch Durchtrennung beider Karotiden and Venae jugulares.

Allgemeinsection: Nirgends Blutungen, auch nicht in Pleura und Endokard.

Schlafenbeine: Beiderseits weder im Mittelohr noch im Labyrinth oder Akustikusstamm Blutergusse.

3. Meerschweinchen, $380 \mathrm{~g}$ schwer. Erhalt $1,0 \mathrm{~g}$ Chinin. mur.; getödtet nach 1/1/2 Stunde, als der Exitus bevorstand. Durchtrennung beider Karotiden und Venae jugulares.

Allgemeinsection: Nirgends Blutungen, auch nicht in Pleura und Endokard. 
Beitrage zur Kenntniss der Wirkung des Chinins auf das Gehororgan. 231

Schlafenbeine: Beiderseits weder im Mittelohr noch $1 \mathrm{~m}$ Labyrinth oder Akustikusstamm Blutergusse.

4. Maus. Erhalt 0,05 g Chmin. mur. Nach 30 Minuten getodtet, als der Exitus bevorstand; analog wie 2 und 3.

Allgemeinsection: Nirgends Blutungen, auch nicht in Pleura und Endokard.

Schlafenbeine: Beiderseits weder im Mittelohr noch $1 \mathrm{~m}$ Labyrinth oder Akustikusstamm Blutergusse.

5. Maus. Erbalt 0,05 g Chinin mur. subcutan. Nach 60 Minuten getodtet, kurz bevor der Exitus zu erwarten; wie die vorhergebenden Versuchsthiere.

Allgemeinsection: Keine Blutungen, auch nicht in Pleura und Endokard.

Schlafenbeıne: Beiderselts weder im Mittelohr noch im Labyrunth oder Akustıkusstamm Blutergusse.

\section{Ir. Durch längere Zeit hindurch verabreichte kleine Dosen vergiftete Thiere.}

1. Kaninchen, $1450 \mathrm{~g}$ schwer. Erhalt taglich $0,3 \mathrm{~g}$ Chinin. mur. Am sechsten Tage eine Stunde nach Application der letzten Dosis Einsetzen schwerer Vergiftungserscheinungen, denen das Thier zu erliegen droht. Kurz bevor der Exitus zu erwarten, Tódtung durch Durchtrennung beider Karotiden und Venae jugulares.

Allgemeinsection: Nirgends Blutungen, auch nicht in Pleura und Endokard.

Schlafenbeine: Beiderseits weder im Mittelohr noch im inneren Ohre oder Akustikus Blutungen.

2. Meerschweinchen, $360 \mathrm{~g}$ schwer. Erhalt taghch $0,025 \mathrm{~g}$ Chinin. mur. Am siebenten Tage 1/2 Stunde nach Application der letzten Dosis Ensetzen schwerer Vergiftungserscheinungen, denen das Ther zu erliegen droht. Todtung wie ber 1 .

Allgemeinsection: Nirgends Blutungen, auch nicht in Pleura und Endokard.

Schlafenbeine: Belderselts weder im Mittelohr noch im inneren Ohre oder Akustikus Blutungen.

Diese letzte Versuchsreihe beweist einwandsfrei, dass auf specifische Chininwirkung zuruckzufuhrende Blutungen in der Regel weder in der Paukenböhle noch im Labyrinth oder Akustikusstainm vorkommen. Blutergusse in der Paukenhöblenschleimhaut bei Thieren, die der Chininintoxication erlagen, mussen, ebenso wie die Petechien in Pleura und Endokard, als agonale aufgefasst werden. Sie sind den 
schweren Suffocationserscheinungen, unter denen die Thiere zu Grunde zu gehen pflegen, zuzuschreiben. In gleicher Weise muss, falls wirklich einmal eine inzweifelhafte Blutung in's Labyrinth oder den Aikustikusstamm nach Chininintoxication beobachtet werden sollte, das Zustandekommen dieser erklärt werden. Aber auch die Annahme, dass das Chinin einen Congestionszustand in Paukenhoble und Labyrinth hervorzurufen im Stande sei, entbehrt nach diesen Untersuchungen jeder sicheren Begründung. Denn es erscheint unzweifelhaft, dass die Erweiterung und starke Fullung der Gefässe in Paukenhöhle und Labyrinth, wie sie Kirchner beschreibt und wie ich sie bei den Versuchsthieren der ersten Versuchsreihe fand, ebenso wie die Blutungen in der Paukenhöhlenschleimhaut, auf agonale Veranderungen, vielleicht auch auf die Tódtungsweise oder dergl. zurückzufüren sind. Da es sich voraussichtlich bei einem durch specifische Chininwirkung unterhaltenen Congestionszustand in Paukenhöhle und Labyrinth um eine rein durch vasomotorische Storung bedingte stärkere Gefässfüllung, und nicht um entzundliche Hyperämie mit Emigration weisser Blutkörperchen, seróser Exsudation und anderen Entzundungserscheinungen einhergehend, handeln würde, so erscheint es unmöglich, einen exacten Nachweis ihres Vorhandenseins oder Fehlens durch eine post mortem angestellte Untersuchung zu erbringen. Denn wir kennen keine Tódtungsart, die eine agonale Veranderung im Füllungszustande der einzelnen Gefässe ausschlösse. Am ehesten wäre dies noch bei Tódtung durch einen elektrischen Schlag denkbar. Immerhin ist selbst dann die Fällung eines Urtheiles ủber den Füllungszustand der Gefảsse durch mikroskopische Untersuchung oft recht schwierig. Auch eine directe Besichtigung der Paukenhöle in vivo nach Entfernung des Trommelfelles verspricht keinen Erfolg, da durch diese Eröffnung schon Circulationsstörungen hervorgerufen werden mússen. Die Untersuchung des Trommelfelles selbst auf der Hobe der Intoxication hat weder beim Menschen ( $G$ ud e r) noch beim Thier (eigene Versuche) constant das Vorhandensein einer auf einen Congestionszustand hindeutenden Injection ergeben.

Wenn wir aber, wie dies sehr nahe liegt, nach Analogie der Veränderungen im Auge Circulationsstörungen auch im Labyrinth in Folge specifischer Chininwirkung annehmen wollen, so wird der Schluss auf eine der Ischämie der Retina entsprechende Ischämie 
Beitrảge zur Kenntniss der. Wirkung des Chinins auf das Gehörorgan. 233 des häutigen Labyrinthes weit berechtigter erscheinen als der auf einen hyperảmischen Zustand, ohne dass wir aber, wie gesagt, hierfür einen sicheren Beweis erbringen können. Mit dieser Annahme stehen weder die klinisehen Beobachtungen noch die experimentellen Untersuchungen am Menschen, noch auch meine eigenen Untersuchungen am Thier, in Widerspruch. 